\title{
THE DYNAMICS OF THE AG CAR RING NEBULA
}

\author{
LINDA J. SMITH \\ Department of Physics and Astronomy \\ University College London \\ Gower Street, London WC1E 6BT \\ England
}

\begin{abstract}
High spatial $\left(2^{\prime \prime}\right)$ and spectral $\left(7 \mathrm{~km} \mathrm{~s}^{-1}\right)$ resolution observations of the ring nebula surrounding the LBV star AG Carinae are presented. The data, covering $\mathrm{H} \alpha$ and [NII], were obtained with the UCL echelle spectrograph on the AAT and cover 4 radial slit positions centred on AG Car. The nebular motions are clearly resolved and show that it is a single shell expanding at $70 \mathrm{~km} \mathrm{~s}^{-1}$ which was ejected from the central star some 7000 years ago. The data also reveal the presence of a high velocity $\left(\sim 83 \mathrm{~km} \mathrm{~s}^{-1}\right)$ bipolar mass outflow which has distorted the northeastern back edge of the shell.
\end{abstract}

\section{Introduction and Observations}

AG Carinae is a member of a small group of stars classified as Luminous Blue Variables (LBV's); other members include $\eta$ Carinae, P Cygni and S Doradus. LBV's are evolved, unstable supergiants which are close to the observed upper luminosity/stability limit in the HR diagram. They are believed to represent a short, unstable phase in the evolution of a massive $O$ star to a WR star. Thackeray (1950) discovered that AG Car is surrounded by a roughly elliptical-shaped $\left(40^{\prime \prime} \times 30^{\prime \prime}\right)$ ring nebula containing two prominent bright clumps to the NE and SW. More recently, circumstellar shells surrounding other LBV's have been discovered (Walborn 1982; Stahl 1989).

The AG Car ring nebula was first observed spectroscopically by Johnson (1976) who found it to be of low excitation because of the lack of [O III] emission. The dynamics were investigated by Thackeray (1977) who obtained photographic spectra of the $\mathrm{H} \alpha,[\mathrm{N} \mathrm{II}]$ and [S II] lines for 9 nebular positions. He detected line splitting $\left(\Delta v \approx 120 \mathrm{~km} \mathrm{~s}^{-1}\right)$ at only one slit position although the data overall indicated that complex motions were probably present in the shell. He derived a rough nebular expansion velocity of $50 \mathrm{~km} \mathrm{~s}^{-1}$. IUE observations by Viotti et al. (1988) revealed that the UV nebular spectrum was very similar to the stellar spectrum, thus indicating the presence of circumstellar dust. Far-infrared observations by McGregor etal. (1988) in a NE-SW direction showed that the dust is cool and is resolved into two peaks which coincide with the bright optical clumps. Paresce \& Nota (1989) imaged the nebula through broad-band optical filters and discovered a prominent bipolar dust structure in the NE-SW direction. In the NE direction, a bright, detached clump of dust is observed whereas in the SW direction, a jet-like feature is seen with a curious helical structure, extending from the star to the edge of the ring nebula. Finally, Mitra \& Dufour 
(1990) present a chemical analysis of the ring nebula; they find that it is oxygen-poor but shows little, if any evidence for a nitrogen enhancement.

The peculiar abundances and the LBV status of AG Car indicate that the ring nebula is composed (at least in part) of stellar ejecta. It is not clear whether the material was ejected as a single shell during a particularly violent mass-loss episode or whether the shell has been formed by the combined action of the stellar wind and multiple, small ejections. For either possibility, it is likely that the strong stellar wind of the progenitor $O$ star evacuated the surrounding interstellar medium. Humphreys et al. (1989) have recently revised the distance to AG Car from $2.5 \mathrm{kpc}$ to $6 \pm 1 \mathrm{kpc}$. This has important consequences for the formation and evolution of the ring nebula since the stellar luminosity is now too high for AG Car to have passed through a red supergiant phase before becoming a LBV. Thus the ring nebula cannot have been formed through the present LBV stellar wind sweeping up material expelled by the red supergiant. The new distance leads to a size of $\sim 1.2 \times 0.9$ pc and a mass of $\sim 2 \mathrm{M}_{\odot}$ (re-scaling the $\mathrm{H} \alpha$ flux given by Stahl (1987)). These revised values are rather interesting since they are now broadly in line with those expected if LBV ring nebulae are the progenitors of some of the ejecta-type WR ring nebulae.

The best means of understanding the origin of the AG Car ring nebula appears to be through a detailed study of the dynamics. For this purpose, high spatial and spectral resolution observations of the ring nebula were obtained with the UCL echelle spectrograph and a GEC CCD detector at the coudé focus of the $3.9 \mathrm{~m}$ Anglo-Australian Telescope (AAT) during 27 April 1989. A slit of dimensions 1 " by $57^{\prime \prime}$ was used with an interference filter to isolate a single order covering $\mathrm{H} \alpha$ and [NII]. Exposure times were between 1000 and $2000 \mathrm{~s}$, sufficient to obtain several thousand counts in the brightest portions of the nebula. The data were bias-subtracted, flat-fielded and wavelength calibrated to an accuracy of 0.3 $\mathrm{km} \mathrm{s}^{-1}$. They have not been absolutely flux calibrated or sky-subtracted (the observations were taken during dark of moon). The spectral resolution of the data is $7 \mathrm{~km} \mathrm{~s}^{-1}$ (as determined from the FWHM of the arc lines) and each spatial increment represents 1.1 arc sec on the sky (the seeing FWHM was 1.7"). H $\alpha$ and [N II] spectra were recorded at four radial slit positions centred on $A G$ Car at position angles $0^{\circ}, 30^{\circ}, 80^{\circ}$ and $131^{\circ}$. Figure 1 shows the exact slit locations superimposed on a [N II] image of the nebula from Stahl (1987) and the corresponding [NII] two-dimensional spectra.

In order to study the nebular kinematics, the [N II] profiles corresponding to each spatial increment were extracted and velocities (relative to the Local Standard of Rest) have been measured for each identifiable nebular component. Velocity maps were then constructed for each slit position and these are shown in Figure 2.

\section{Results and Discussion}

The spectra displayed in Figure 1, together with the velocity maps given in Figure 2, show obvious nebular line splitting. Overall, they broadly show that the ring nebula is a complete hollow, expanding shell of non-uniform brightness. (The $\mathrm{H} \alpha$ spectra are essentially identical and will not be discussed here because their velocity resolution is lower due to larger thermal broadening.) The most remarkable features observed in the [N II] spectra are two high velocity structures indicative of a bipolar mass outflow. These features are most striking for P.A. $=0^{\circ}$ where two oppositely opposed high velocity streams of equal brightness are seen extending from near the star to beyond the edge of the shell. The positive velocity feature is $\sim 19^{\prime \prime}$ long with $V_{L S R}=+90 \pm 6 \mathrm{~km} \mathrm{~s}^{-1}$ and the negative velocity counterpart is 
of similar length with $V_{L S R}=-77 \pm 2 \mathrm{~km} \mathrm{~s}^{-1}$ (the lower velocity means that it is blended with the approaching side of the shell near the star so is less easily recognisable). The positive velocity counterpart is also seen at P.A. $=30^{\circ}$ with $V_{L S R}=+84 \pm 6 \mathrm{~km} \mathrm{~s}^{-1}$ and a spatial extent of $\sim 14^{\prime \prime}$. Both high velocity features are also just visible in the data for P.A. $=131^{\circ}$ where two faint structures $4^{\prime \prime}$ long can be seen close to the star with slightly higher velocities of $-102 \pm 2$ and $+99 \pm 4 \mathrm{~km} \mathrm{~s}^{-1}$.

The angular extent of these features over the nebula seems to be quite small since they are only both fully seen at P.A. $=0^{\circ}$ and $180^{\circ}$. The fact that the positive velocity counterpart is only seen at P.A. $=30^{\circ}$, together with the fact that for P.A. $=131^{\circ}, 311^{\circ}$ the two features have a very small spatial extent, leads to an approximate upper limit to the angular size of $30^{\circ}$. There appears to be no obvious corresponding features on the [NII] image in Figure 1. Whether they correspond to the bipolar dust features seen by Paresce $\&$ Nota (1989) is less clear. The slit position for P.A. $=30^{\circ}, 210^{\circ}$ crosses the minor axis of the nebula, passing through the detached dust clump (P.A. $=23^{\circ}-31^{\circ} ; 9.5^{\prime \prime}$ from the star) and grazing the dust jet (P.A. $=210^{\circ}-230^{\circ}$, ending at $15^{\prime \prime}$ from the star). From this, the positive velocity feature extending from $0^{\circ}-30^{\circ}$ encompasses the dust clump but the negative velocity feature is absent at the position angle of the jet. In addition, the high velocity features cover a much greater spatial extent as they extend beyond the shell.

If we assume that the high velocity features detected at P.A. $=0^{\circ}, 180^{\circ}$ are moving away from the star at the same velocity, the mean velocities given above lead to a projected expansion velocity of $83 \mathrm{~km} \mathrm{~s}^{-1}$ centred on a systemic velocity of $+7 \mathrm{~km} \mathrm{~s}^{-1}$, in remarkable agreement with the stellar radial velocity of $+7 \mathrm{~km} \mathrm{~s}^{-1}$ (Wolf \& Stahl 1982). We conclude that the high velocity features can be interpreted in terms of a jet-like bipolar mass outflow with a projected velocity of $83 \mathrm{~km} \mathrm{~s}^{-1}$ which is not clearly related (at least at this epoch) to the bipolar dust features of Paresce \& Nota (1989).

Turning to the dynamics of the shell itself, the data in Figures 1 and 2 indicate that it is basically expanding away from the star with some distortions from spherical symmetry. These distortions are most evident for the slit at a position angle of $30^{\circ}$. The brightest parts, defining the shell, appear to be symmetrical with respect to the central star, both spatially and kinematically. The north-eastern quadrant, where the material is moving away on the far side of the nebula, is much fainter with higher velocities and a greater spatial extent. This part of the slit corresponds to the faint material which is visible beyond the brightest portion of the ring in the [NII] image shown in Figure 1. Both the dynamical and image information therefore suggest that at this position angle, the shell is expanding more rapidly away from the star. This spatial and velocity discontinuity is also seen, to a lesser extent, at P.A. $=0^{\circ}$. The high positive velocity counterpart of the bipolar mass outflow (discussed above) is also observed at these two position angles, as well as the dust clump at P.A. $=30^{\circ}$ (Paresce \& Nota 1989). This coincidence suggests that the observed distortion of the shell can be explained if the higher velocity outflow has pushed against the shell and accelerated it.

Images of the ring nebula (cf. Figure 1) show that it is slightly elliptical in shape. The shell should therefore to be an ellipsoid, orientated at some angle to the line of sight, rather than a simple sphere. Some ellipticity does appear to be present since a velocity tilt is observed in the data for P.A. $=0^{\circ}$ with the upper edge of the shell red-shifted compared to the lower edge. The slit at P.A. $=131^{\circ}$ crosses the major axis of the nebula on the sky and shows no obvious velocity tilts. The slit position for P.A. $=80^{\circ}$ presents the simplest nebular geometry overall since the back and front of the expanding shell appear to be roughly spherically symmetric. Taken as a whole, the data for the four slit positions 



Figure 1: Two-dimensional [N II] spectra covering the four slit positions shown in the [N II] image of the AG Car ring nebula (reproduced with permission from Stahl (1987)). The intensity scale for the spectra is logarithmic; a stellar occulting mask was used for P.A. $80^{\circ}$. 

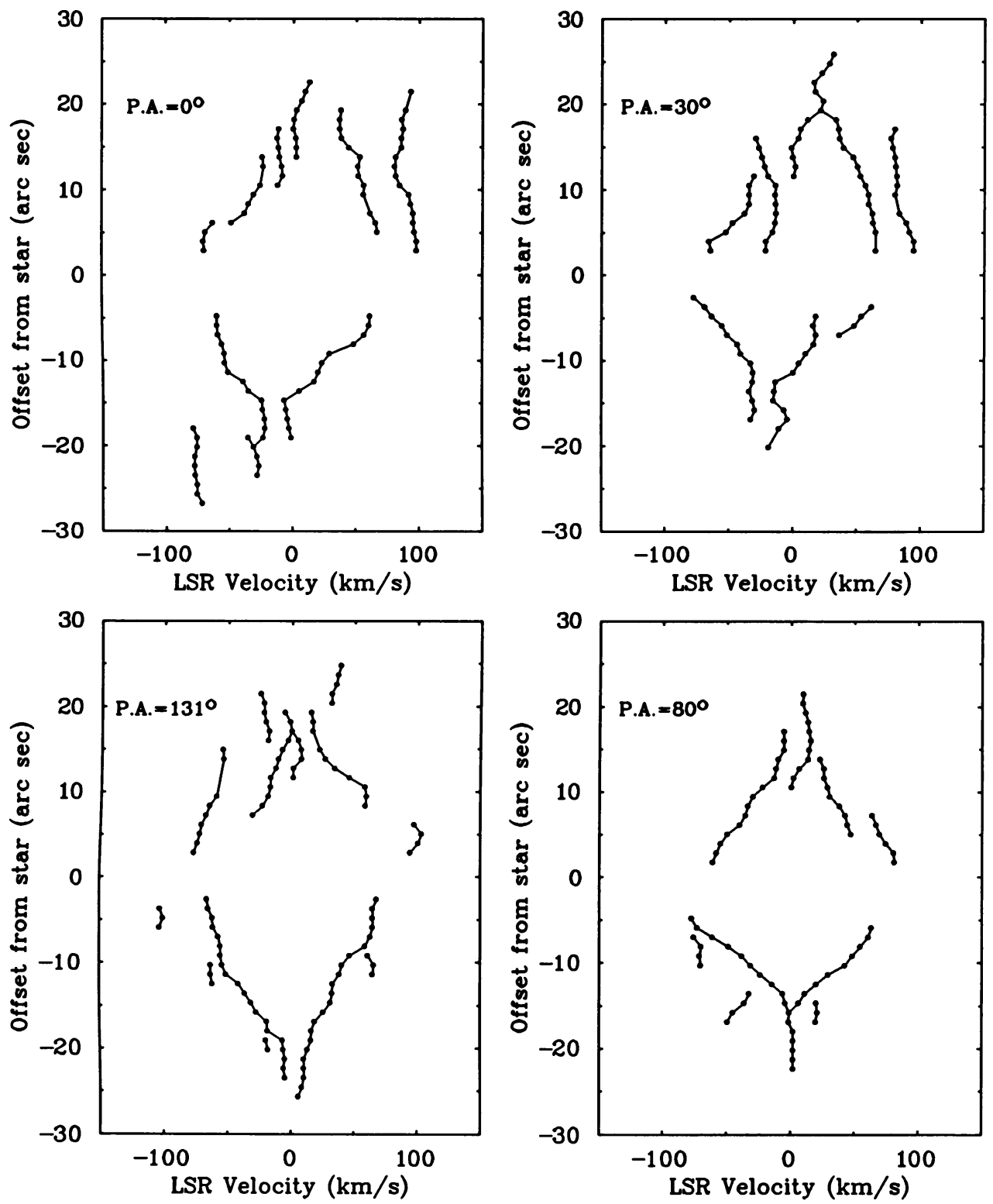

Figure 2: Measured LSR velocities of identified features in the four [N II] images plotted against the offset in arc sec from AG Car. Recognised continuous features in velocity space are depicted by solid lines. 
show broadly similar velocity patterns; this overall uniformity suggests that the ring nebula resulted from a single ejection of material from AG Car.

Regarding the expansion velocity of the shell, the velocity maps in Figure 2 (particularly those for P.A. $=80^{\circ}$ and $131^{\circ}$ ) indicate that the shell expansion is roughly symmetric and centred on a velocity of $0 \mathrm{~km} \mathrm{~s}^{-1}$. The shell expansion velocity is then directly given by the observed approaching and receding velocities at the position of the star; these are measured to be $\pm 70 \mathrm{~km} \mathrm{~s}^{-1}$. Additional evidence in support of this expansion velocity is provided by the interstellar NaI absorption lines towards the central star AG Car. Data obtained at the same resolution as the nebular spectra reveal an absorption component at $V_{L S R}=-67 \mathrm{~km} \mathrm{~s}^{-1}$ which is also seen in Ca II at the same velocity by Wolf \& Stahl (1982). The derived expansion velocity is substantially higher than the value of $50 \mathrm{~km} \mathrm{~s}^{-1}$ quoted by Thackeray (1977) and leads to a dynamical age, $t_{D} \approx R / V_{\exp } \approx 7 \times 10^{3} \mathrm{yr}$ for the AG Car ring nebula (assuming that the radius $R=0.5 \mathrm{pc}$ ). Over this timescale, the stellar wind of AG Car will have contributed at most $\sim 0.5 \mathrm{M}_{\odot}$ to the nebular mass of $2 \mathrm{M}_{\odot}$ if the upper limit of $7 \times 10^{-5} \mathrm{M}_{\odot} \mathrm{yr}^{-1}$ is adopted for the stellar mass loss rate (Lamers 1989). The shell is unlikely to contain any swept-up interstellar material because the progenitor $O$ star will have evacuated the surrounding interstellar medium. The amount of stellar material ejected by AG Car $7 \times 10^{3}$ yr ago is therefore $\sim 1.5 \mathrm{M}_{\odot}$.

\author{
References \\ Humphreys, R.M., Lamers, H.J.G.L.M., Hoekzema, N. \& Cassatella, A., 1989. \\ Astr. Astrophys., 218, L17. \\ Johnson, H.M., 1976. Astrophys. J., 206, 469. \\ Lamers, H.J.G.L.M., 1989. in Physics of Luminous Blue Variables, eds. K. Davidson, \\ A.F.J. Moffat \& H.J.G.L.M. Lamers, Kluwer, Dordrecht, p.135. \\ McGregor, P., Finlayson, K., Hyland, A.R., Joy, M., Harvey, P.M. \& Lester D.F., \\ 1988. Astrophys. J., 328, 874. \\ Mitra, P.M. \& Dufour, R.J., 1990. Mon. Not. R. astr. Soc., $242,98$. \\ Paresce, F. \& Nota, A., 1989. Astrophys. J., 341, L83. \\ Stahl, O., 1987. Astr. Astrophys., 182, 229. \\ Stahl, O., 1989. in Physics of Luminous Blue Variables, eds. K. Davidson, \\ A.F.J. Moffat \& H.J.G.L.M. Lamers, Kluwer, Dordrecht, p.149. \\ Thackeray, A.D., 1950. Mon. Not. R. astr. Soc., 110, 524. \\ Thackeray, A.D., 1977. Mon. Not. R. astr. Soc., 180, 95. \\ Viotti, R., Cassatella, A., Ponz, D. \& Thé, P.S., 1988. Astr. Astrophys., 190, 333. \\ Walborn, N.R., 1982. Astrophys. J., 256, 452. \\ Wolf, B. \& Stahl, O., 1982. Astr. Astrophys., 112, 111.
}

\title{
DISCUSSION
}

Walborn: Is there any evidence for chemical composition differences among the various velocity features in your data?

Smith, Linda: I only have $H \alpha$ and $[N I I]$ data and have not examined the $[N I I] / H \alpha$ ratio in any detail. The appearance of the $H \alpha$ and $[N I I]$ two-dimensional spectra are very similar, indicating that the various velocity features show no obvious differences in the $[N I I] / H \alpha$ ratio.

Schulte-Ladbeck: I am wondering about the geometry, i.e., the position angle of the bipolar outflow with respect to the Paresce features. Are they perhaps perpendicular?

Smith, Linda: No, they have similar position angles. They are, however, perpendicular to the major axis of the ring nebula, which is probably significant. 